\title{
Endoscopically Placed Rectourethral Guidewire Facilitates the Reconstruction of Anus in Children with Anorectal Malformations: A Case Report
}

\author{
Pernilla Stenström ${ }^{1}$ Magnus Anderberg ${ }^{1} \quad$ Christina Clementson Kockum ${ }^{1}$ Einar Arnbjornsson ${ }^{1}$ \\ ${ }^{1}$ Department of Pediatric Surgery, Skane University Hospital, Lund, \\ Address for correspondence Einar Arnbjornsson, MD, PhD, Associate \\ Sweden \\ Professor, Department of Pediatric Surgery, Skane University Hospital, \\ Eur J Pediatr Surg Rep 2013;1:46-47. \\ Lund 22185, Sweden (e-mail: einar.arnbjornsson@telia.com).
}

\begin{abstract}
Keywords

- anorectal malformation

- posterior sagittal anorectoplasty

- newborns

- endoscopy

- rectourethral fistula

The aim of this report is to present a technical detail of use in the management of an anorectal malformation (ARM). A boy with ARM and a fistula to the urethra was operated on with a posterior sagittal anorectoplasty (PSARP). Before the PSARP operation, a videoendoscopy was performed through the distal stoma down to the distal end of the rectum. The rectourethral fistula was identified and a guidewire was passed through the endoscope, through the fistula, and out through the urethra. The endoscopically placed rectourethral guidewire was used as a landmark and facilitated the PSARP operation.
\end{abstract}

\section{Introduction}

When reconstructing an anorectal malformation (ARM) with posterior sagittal anorectoplasty (PSARP), the dissection of the rectourethral fistula is one of the challenges. The PSARP procedure includes opening the rectum posteriorly to clarify the anatomy before dividing the rectourethral fistula. ${ }^{1}$ The aim of this report is to describe an approach including placing of a guidewire passed through the endoscope in the colostomy, through the fistula to the urethra to facilitate the localization and dissection of the rectourethral fistula early in the operative intervention.

\section{Case Report}

A boy was born in the 38th gestational week, with a birth weight of 2,980 g. On examination, he was found to have an anal atresia without fistula to the perineum. No other congenital malformations were discovered.

At the age of 20 hours postpartum, the boy was provided with a left loop divided colostomy by a laparoscopic approach. At the age of 6 weeks, the boy was scheduled for a PSARP

received

February 16, 2013

accepted after revision

March 15, 2013

published online

April 24, 2013

operation. Preoperative colon X-ray examination disclosed the open rectourethral fistula in the distal end of the rectum.

Before the PSARP operation, an endoscope was inserted through the distal stoma and the fistula to the urethra was identified at the distal end of the rectum. A guidewire was passed through the endoscope into the fistula and out through the urethra (-Figs. 1 and 2). A catheter was placed into the urinary bladder in the traditional way through the urethra. Video endoscope of $6.5 \mathrm{~mm}$, a guidewire of $2.5 \mathrm{~mm}$, and bladder catheter of 6 Charrière were used.

The PSARP operation was performed. The dissection of the rectourethral fistula was facilitated by the guidewire in the fistula. The fistula could initially be shown and dissected without opening the rectum and the guidewire was visualized on opening the distal end of the rectum. Surgical damage to surrounding tissues and intestine was thereby avoided by using this artificial benchmark of the fistula. No dissection in search of the rectourethral fistula was needed. During the PSARP operation, the fistula was divided and used for reconstructing the new anus, while the opening to the urinary tract was left without suture. ${ }^{2}$

C 2013 Georg Thieme Verlag KG
Stuttgart · New York
DOI http://dx.doi.org/ $10.1055 / \mathrm{s}-0033-1345104$. ISSN 2194-7619. 


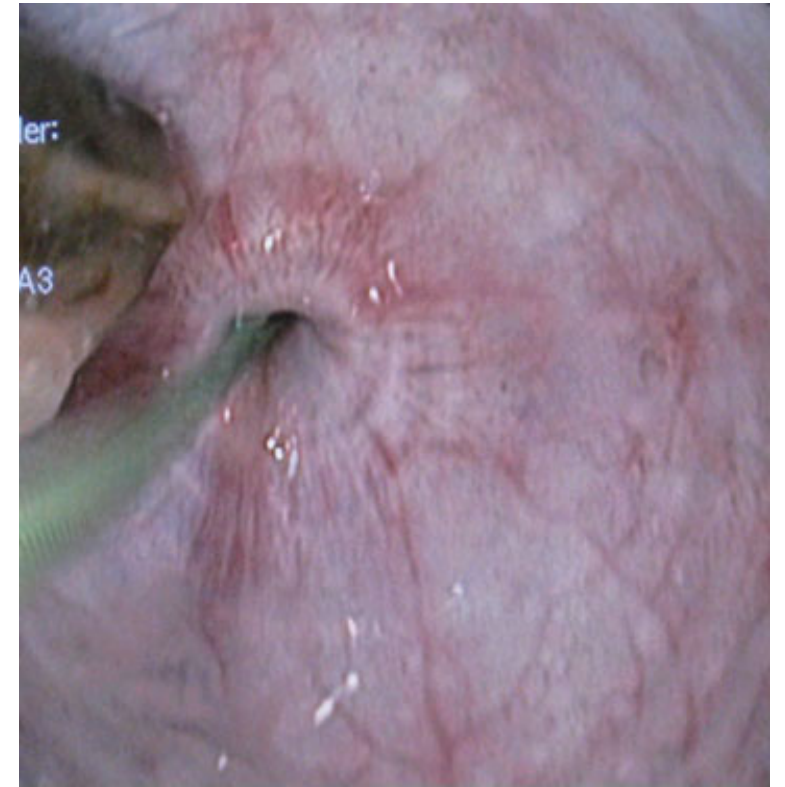

Fig. 1 Through the videoendoscope the rectourethral fistula is easily identified and a guidewire is passed through the videoendoscope and through the rectourethral fistula.

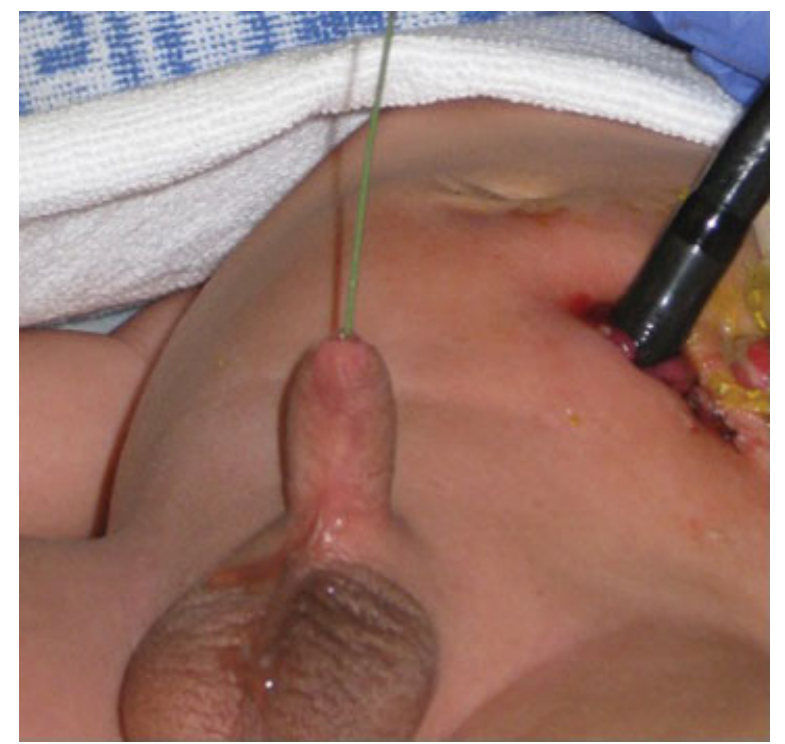

Fig. 2 Through the endoscope of $9 \mathrm{~mm}$, a guidewire of $2.5 \mathrm{~mm}$ has been passed through the rectourethral fistula.

The postoperative course was uneventful. An X-ray of the urinary bladder and urethra, voiding cystourethrogram, performed 6 weeks later showed a normal urinary tract without any fistula to the bowel.

\section{Discussion}

This case report demonstrates the use of an endoscopy-aided placement of a rectourethral guidewire to facilitate the reconstruction of anus in patients born with ARM. Reliable placement of a catheter in the fistula through the urethra is impossible. The placement of a guidewire from the distal end of the rectum to the urethra was found to be easy.

A guidewire through the fistula facilitates the dissection and thereby helps avoiding any unnecessary dissection searching for the rectourethral fistula. The anatomy becomes clearer, and with the wire in place, opening of the intestine during the initial part of the dissection is prevented. By using the guidewire, the leveling of the division or the fistula is facilitated. Any suspicion of nonexisting fistula could be disregarded through the view from the endoscope.

With the video endoscope introduced through the distal stoma, it is usually easy to identify the rectourethral fistula preoperatively. The risk of damage to the urethra may be reduced and that might lead to fewer problems with urethral stricture or urethral diverticula. With more exact dissection, it is easier to save the tissues in the fistula for reconstruction of the anus. Furthermore, the air in the bowel introduced through the endoscope facilitates the dissection of the blindending bowel.

Endoscopy-assisted laparoscopic excision of rectourethral fistula in a male patient with imperforate anus has been described without the use of any guidewire. ${ }^{3}$ By using the method described here, laparoscopy/laparotomy intervention in the abdomen is facilitated.

To summarize, we have found that the method described here has facilitated the PSARP intervention. Since the first time this operative intervention described here was performed, we have used the method in several children with ARM with rectourethral fistula, including a child with cloaca. When performing these interventions, we suggest that both options for placing a guidewire through a cystoscopy from the urethra or from a video endoscope from the bowel should be available. We hereby submit the method for evaluation by others.

\section{Acknowledgment}

The authors are indebted to Gillian Sjödahl, Lexis English for Writers, Lund, Sweden, for linguistic revision of the manuscript.

\section{Conflict of Interest}

None

\section{References}

1 Peña A. Anorectal malformations. Semin Pediatr Surg 1995;4(1): 35-47

2 Rollins MD, Downey EC, Meyers RL, Scaife ER. Division of the fistula in laparoscopic-assisted repair of anorectal malformations-are clips or ties necessary? J Pediatr Surg 2009;44(1):298-301

3 Yamataka A, Kato Y, Lee KD, Lane G, Kusafuka J, Okazaki T, et al. Endoscopy-assisted laparoscopic excision of rectourethral fistula in a male with imperforate anus. J Laparoendosc Adv Surg Tech A 2009;19(Suppl 1):241-243 